\title{
CHARGE syndrome
}

\author{
A M M K Athapaththu', N M Koswatta ${ }^{2}$ \\ Sri Lanka Journal of Child Health, 2013; 42(2): 101-102
}

(Key words: CHARGE syndrome; choanal atresia; coloboma)

\section{Introduction}

CHARGE (Coloboma of eyes, heart defects, atresia choanae, retardation of growth and development, genital anomalies, ear anomalies) syndrome is a nonrandom association, typically caused by mutations in CHD 7 gene $^{1}$. Prevalence is $1: 10,000^{2}$. It affects both sexes equally and is inherited in an autosomal dominant pattern ${ }^{1,3}$.

\section{Case report}

A primigravida with a non consanguineous marriage and polyhydramnios had an emergency caesarean section at term due to fetal distress and delivered a baby boy. He did not cry at birth and recovered with bag and mask ventilation. APGAR scores at one, five and ten minutes were 3,7 and 8 respectively. Respiratory distress persisted. Nasogastric (NG) tube did not pass through both nostrils.

On examination, he was tachypnoeic, with intercostal and subcostal recessions, having simple ears, right hand polydactyly and cryptorchidism (Figures 1 \& 2). A presumptive diagnosis of CHARGE syndrome was made. He was ventilated in first hour of age due to worsening of respiratory distress and supportive treatment with antibiotics was commenced. CT scan of nose and paranasal sinuses showed bilateral choanal atresia, bony type (Figure 3 ).

Drilling and stenting was done on day 2 of age by the paediatric ENT team. 2D echocardiography showed ostium secundum atrial septal defect, moderate size patent ductus arteriosus and tetralogy of Fallot. Both eyes were found to have disc colobomas. Ultra sound scan (USS) of abdomen showed no evidence of renal anomalies. USS of brain showed prominent ventricular system with cisterna magna. He was

${ }^{1}$ Medical Officer Neonatology, ${ }^{2}$ Consultant
Neonatologist, Teaching Hospital, Kandy

(Received on 17 September 2012: Accepted after revision on 19 October 2012) ventilated for 6 days and was on continuous positive airway pressure for 2 days. On day 10 he developed tachypnoea and as chest x-ray showed aspiration pneumonia he was re-started on antibiotics. On discharge on day 36 of age he was starting to gain weight but was still on NG feeds.

\section{Discussion}

CHARGE syndrome is a clinical diagnosis and some clinical features have been proposed as criteria in diagnosis $^{2}$. Structural defects in CHARGE syndrome are attributed to arrest in embryonic differentiation in the second month of gestation (choanae at 35-38 days, cochlea at 36 days, cardiac septum 32-38 days, eye at 5 weeks, and external ear at 6 weeks of gestation).

Presentation of CHARGE syndrome can be intrauterine (Intra uterine growth retardation, poor fetal movements, congenital heart defects), neonatal (small for gestational age, dysmorphic features, NG tube not passing, feeding difficulty, respiratory distress) or infantile/childhood (failure to thrive, developmental delay, feeding difficulty). No single clinical feature is present universally in CHARGE syndrome $^{1}$. Colobomas of eyes are usually bilateral. Septal defects and conotruncal malformations are the most common heart defects. Choanal atresia is bilateral in $50 \%$ and could be membranous or bony in nature ${ }^{1}$. Growth retardation is found in $80 \%{ }^{1}$. Genital hypoplasia (males: micropenis, cryptorchidsm or complete absence of penis; females: labial hypoplasia) is more common in males. Ear malformations consist of small, simple, low set or cup shaped ears. Hearing loss is usually bilateral and mixed type in nature. Neurologic anomalies (facial nerve palsy, microcephaly), mental retardation, facial dysmorphism (asymmetric square face, micrognathia, malar flattening) are also found. Imaging studies are done to see the structural defects. Surgical and medical problems are managed accordingly. 


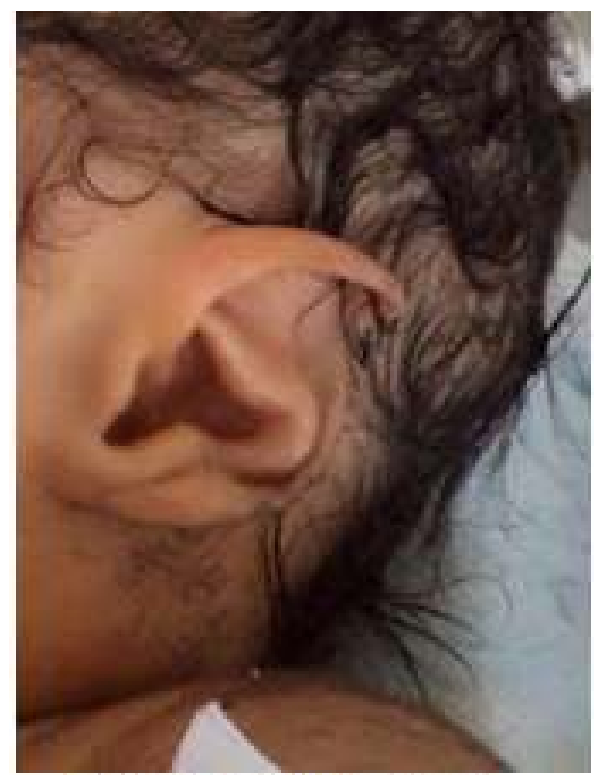

Figure 1: Simple ears

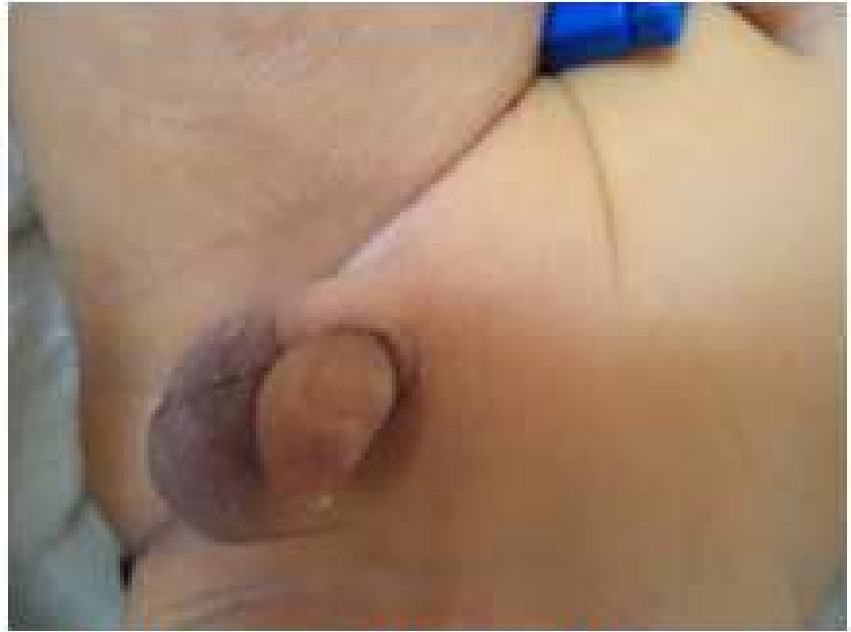

Figure 2: Cryptorchidism
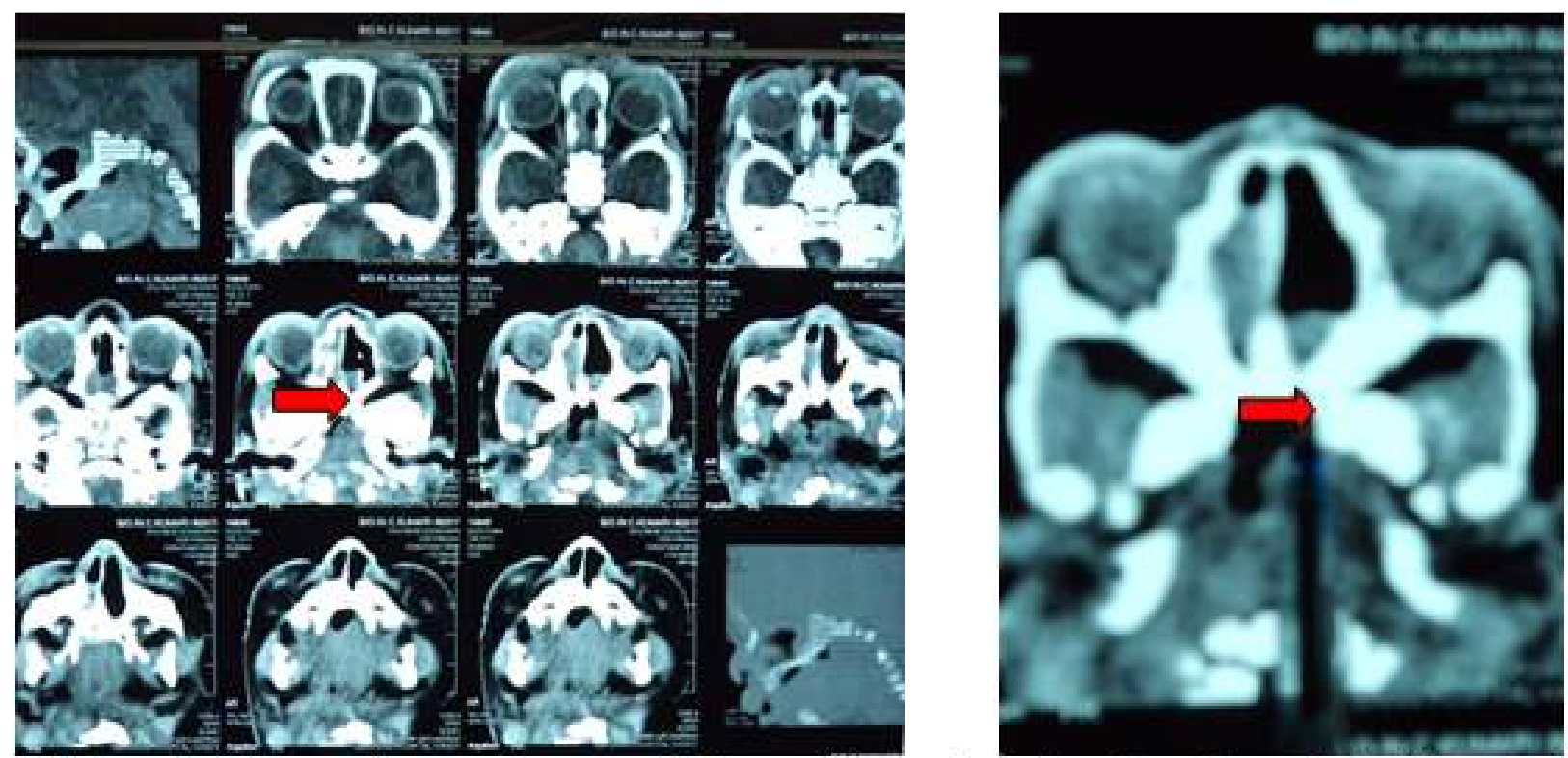

Figure 3: CT scan of nose and paranasal sinuses (Arrow shows bony type choanal atresia)

\section{References}

1. Tegay DH. CHARGE Syndrome: Medscape Reference Drugs, Diseases \& Procedures; 2012 [cited 2012 Mar 12].Available from: http://emedicine.medscape.com/article/942350-overview.

2. Blake KD; Davenport SL; Hall BD; Hefner MA; Pagon RA; Williams MS; et al. CHARGE association: an update and review for the primary paediatrician. Clinical Pediatrics (Phila) 1998; 37(3):159-73.

3. CHARGE Syndrome: Genetics Home Reference; U.S. National Library of Medicine; 2008 May [cited 2012 Jul 30]. Available from: http://ghr.nlm.nih.gov/condition/charge-syndrome 